\title{
Roles of a Center and Institute in Promoting Regional Research Collaborations
}

\author{
Peter G. Smith
}

Director, Institute for Neurological Disorders

Co-Director, Kansas Intellectual and Developmental Disabilities Research Center

Professor, Molecular and Integrative Physiology, University of Kansas Medical Center

$\mathrm{T}$

The role of collaborations in research has become more dominant with the passage of time. This should not be surprising given the evolution of the study of biomedical problems. If we look back to the middle of the last century, research was very different. Problems were more basic - we had just begun to recognize and understand the genome and molecular biology was yet to be invented; the tools for research were relatively rudimentary - the electron microscope, which ultimately revolutionized cell biology, was just beginning to enjoy widespread use; and the questions investigators asked were very much framed by their discipline; most researchers were trained in a single area, and applied a single technique to just one aspect of a problem. The pressure to pursue an integrated approach was minimal.

This began to change rapidly in the latter part of the $20^{\text {th }}$ century. Problems became more complex, with larger available armamentaria with which to pursue these questions. Of course, with more advanced technologies and a greater desire to incorporate multidisciplinary approaches in attacking biomedical problems, also came challenges. Training began to lag behind technology; it became difficult to find individuals whose breadth of technical skills was suitable to bring to bear upon the full range of emerging medical questions. Clearly, the easiest way to achieve the necessary critical mass of technologies and disciplines was through research collaborations.

While a need for creating research collaborations has been recognized for some time, the magnitude of collaborations necessary for advancing biomedical research has continued to grow with the increasing complexities of the questions at hand. We have seen the norm in academic collaborations grow from simple ad hoc associations that develop in a grass-roots manner, to the creation of research teams in more formal settings. While this model is widely applied in the commercial and government sectors, it has been late in coming to academia, owing to several reasons. In large part, this may be attributed to a number of logistical issues that have to be overcome in order to successfully develop collaborative 
research teams. Some of these issues are as follows.

- Identifying target areas. There need to be clearly defined and agreed upon target areas that will provide a framework in which collaborative research programs can develop and grow.

- Growing the investigator base. In order to attain meaningful collaborative teams, it is essential to have sufficient strength to draw upon so that teams with the appropriate expertise are in place. This may involve either identifying existing potential members, or recruiting members to join the sponsoring institution

- Creating group cohesion and a common cause. A collaborative group needs to see a common vision. If the programmatic direction is unclear, if the vision is unshared, or if the outcome of the collaboration is vague or in dispute, collaborative research will suffer. However, mechanisms must also be in place to permit adjustments to programmatic direction that take into account changes in strength due to the addition of new members or the loss of existing members.

- Thinking regionally. Given the range of approaches that can be potentially applied to biomedical research problems, it is becoming more likely that collaborations beyond the walls of any given academic institution become necessary; this is especially true of smaller institutions. These types of collaborations raise a new set of issues; bridging institutional barriers, distribution of resources, and overcoming problems associated with distance between institutions are chief among these.

How can we encourage development of regional collaborative research enterprises? There are probably a large number of potential approaches, and there clearly is no absolute formula that can guarantee success. However, we have been fortunate in having some success in developing collaborative programs, and our model may be instructive to others who may wish to replicate the experience - or perhaps learn from our mistakes!

Role of a Research Center in $\underline{\text { developing collaborations }}$

The University of Kansas was fortunate in 1966 to be one of an elite handful of universities to be awarded funds from the newly formed National Institute for Child Health and Human Development (NICHD) to create a Mental Retardation Research Center (MRRC). KU, under the leadership of Richard Schiefelbusch, was one of twelve original host sites for sponsoring these MRRCs. The objective of these centers was to promote research in mental retardation and other disabilities affecting the nervous system and behavior. While the objective of this NIH P30 grant was not to initiate research collaborations per se, the award did a number of important things in easing us along this path. One factor was that it required us to identify and develop research themes. Each center is expected to have areas of research emphasis relevant to mental retardation and developmental disabilities (MRDD). In our case, we began with a substantial number - originally in excess of 8 . What is somewhat surprising is that, rather than increasing the numbers of themes over time as might be expected to occur with normal institutional growth, the number actually decreased. It appears that some Darwinian processes may be 
at hand, favoring the perpetuation of some themes and the demise of others.

There may be several reasons for this. One is clearly related to leadership. Those themes that had strong leaders remained viable, while those where a clear leader was less obvious did not prove to be durable. A second factor appears to be related to group drift. Stronger groups, while perhaps not intentionally meaning to, often times absorb members of the weaker groups. This may be associated with people gravitating to the stronger leaders, but is also seems that thriving programs may provide greater opportunities - and some of these are in the form of potential collaborations. Because of a need to define themes, the MRRC grant formalized and legitimized potential collaborative areas and identified and empowered group leaders to move these areas forward. So while the major thrust of the MRRC grant was not to develop research collaborations per se, it was probably inevitable that this should have been the case.

These principles seem aptly illustrated with regard to the evolution of the R.L. Smith Research Center, the Kansas City branch of the MRRC (the component with which I am most familiar). A new building constructed for the purpose of housing MRRC programs in common space (although that capacity was rapidly exceeded) opened in 1972. In the initial brochure describing Smith Center, 6 thematic areas were listed.

" Reproductive physiology \& Neuroendocrinology

» Human Genetics

» Developmental Physiology
» Impaired fetal \& infant development

» Neurobiological mechanisms

» Educational and Pediatric psychology

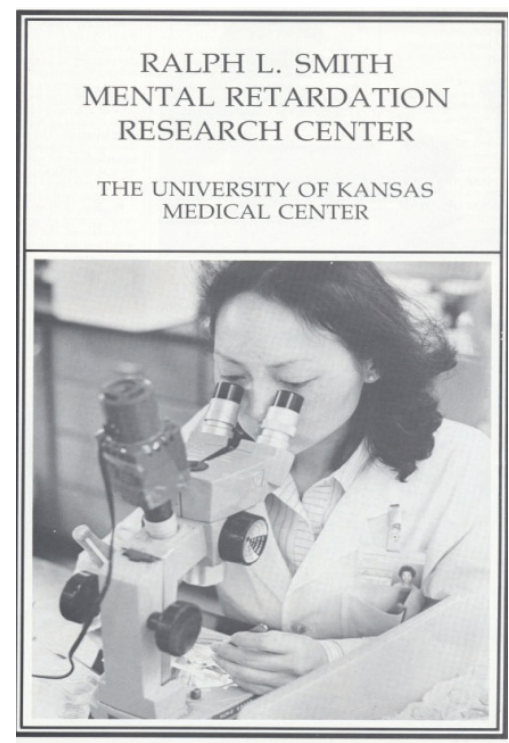

Through the pressures described above, the fates of these areas changed over the next decade and a half. Thus, by the mid-1980's, the number of thematic research areas had diminished essentially to 2: biology of early development (reproductive biology) and neurobiology of intellectual disabilities.

Why did this occur? One probable reason is that maintaining so many divisions requires considerable effort. Another is that not all areas had the mass necessary to sustain (some likely created to induce participation by catering to territorial wants, thus slicing the pie rather thinly). In some cases, key individuals left for other positions. One factor accounting for the durability of the two surviving themes was that they were inherently more inclusive, ably accommodating many members of the smaller original groupings. And perhaps most importantly, both remaining 
divisions were able to identify strong leaders who were able to consolidate groups of researchers. Gilbert Greenwald, chair of physiology, was able to bring together and organize a growing number of reproductive biology researchers and, similarly, Fred Samson, Director of the Smith Center, served in a similar role in promoting neurosciences. Both of these leaders were in positions to effect recruitments in their respective areas, thus increasing numbers of collaborating researcher, as well as in organizing existing faculty.

While our original organizers clearly played critical roles, the importance of continuity in leadership should not be underestimated. In both cases, when the time came to pass the mantle, there were relatively clear lines of ascension, with Paul Terranova (now vice chancellor for research) stepping in to lead the reproductive sciences group, and Paul Cheney (now chair of molecular and integrative physiology) assuming direction of the Smith Center and representing the neurosciences group. Continued group cohesion is highly dependent on having potential incumbent leaders in the wings that are able to step in when conditions dictate.

As a result of the activities of the MRRC, research on the KUMC campus was impacted very significantly by laying down groundwork for organized research collaborations in 2 areas that have persisted over the years. And indeed, over the years the payoffs have been substantial. These are probably best illustrated in the area of reproductive sciences. In the past decade or so, there have been several program project grants that have come out of the reproductive sciences group. Very significantly, we have seen center grants (initially a P30 and subsequently a U54) in reproductive sciences and now in male contraception come directly from this group, as well as an Institute for Maternal and Fetal Biology. Thus, in the case of reproductive sciences, the MRRC served as an incubator in which a number of newer sub-themes reemerged, building on the collaborations that were encouraged by the structure of the Mental Retardation Research Center grant (see article by Paul Terranova, $p$. 5).

Role of an Institute in developing regional research collaborations

The successes of the MRRC (subsequently renamed the Eunice $\mathrm{K}$. Shriver Kansas Intellectual and Developmental Disabilities Research Center [KIDDRC] in 2007) have been substantial and impressive. However, there were some limitations that, in the evolving environment, are likely to have impeded regional research collaborations. While the NICHD center grant (P30) mechanism was essential in encouraging the establishment of core groups in reproductive biology and neuroscience at KUMC, the ground rules, by their very nature, also limited the evolution of these groups.

Centers funded by the NIH are intended to serve a specific purpose. In the case of the IDDRCs, they are intended to "...advance the diagnosis, prevention, treatment, and amelioration of intellectual and developmental disabilities." (http://grants.nih.gov/grant s/guide/rfa-files/RFA-HD-08-016.html).

This requirement immediately places constraints on the types of research that 
can be included in the IDDRC portfolio. Further, membership within the IDDRC research themes is not open to all investigators. Thus, the purpose of the IDDR center grant is to provide core technical, scientific and administrative support investigators with funded research programs related to forms of intellectual and/or developmental disabilities. Because this is the mission of the NICHD and this Institute is footing the bill, it is totally appropriate that activities be selectively supported. But this also places restrictions on the types of interactions that can occur. Thus, interactions with investigators who have related or complimentary funded research programs, but which are not ostensibly relevant to IDD, are not encouraged by this model. Similarly, those with programs that are relevant but not currently funded are also not supported. Further, because the center is based on a single-institution model, individuals who have relevant programs within the region but outside of the parent institution are not encouraged to participate. And finally, because many clinicians with interests and patient populations relevant to IDDRC thematic areas lack requisite funding, interactions with these clinician-investigators are impeded by the P30 model. While there is a strong rationale for having these guidelines in place, it is also clear that these strictures can impede a research center from evolving to the next level of being a base for regional clinical and basic biomedical research.

How can these limitations be circumvented? The need for alternative strategies to broaden the collaborative research base has been an issue of particular relevance to neuroscientists at the University of Kansas Medical Center. Basic neuroscience research has been a powerhouse at KUMC for some time, owing much of its success to the organizational framework created by the MRRC. In 2008, KUMC had some 40 scientists with programs related to the neurosciences, and with a funding portfolio of approximately $\$ 70 \quad \mathrm{M}$. Perhaps another 80 basic scientists and clinicians were present at KUMC or regionally. Yet the Neurobiology theme of the IDDRC at this time included only 19 members! Clearly, there was a strong need to create an alternative infrastructure that would be more inclusive if we wanted to foster stronger interactions among basic and clinical researchers in the neurosciences. This led to the conceptualization of a regional entity to better accommodate collaborations: the Institute for Neurological Disorders (IND).

The decision to move forward with the IND represented a convergence of multiple factors. An important component was a strategy on the part of KUMC administration to more clearly articulate its priorities and goals for the next 10 years. The result of that effort was a document entitled "The Time is Now" (http://www.kumc.edu/evc/TheTi meIsNow.pdf), in which existent strengths in the neurosciences were acknowledged, making this discipline one of the top priorities. In response to a need to better organize the neuroscience effort, departmental chairs and center directors in programs relevant to the neurosciences convened and formed a plan to create the Institute for Neurological Disorders. Several features 
of the IND were put in place to maximize collaborative output.

- A regional entity; no walls. An important aspect of the IND is that, while it is based at KUMC, all regional neuroscientists can be members and participate. This recognizes not only the fact that the Kansas City neuroscience community exists in many relatively small institutions, each lacking desirable critical programmatic mass, but also that in many instances such collaborations had already been established at the grass roots level.

- A place for clinicians as well as basic sciences. In the current atmosphere where the highest value is placed on 'translational' activities, it is essential that the full range of biomedical activities, from discovery to application, be represented. Accordingly, it is important to include members in both the clinical and basic sciences arenas.

- Organization by discipline; finding common threads. Neuroscience is an extremely broad field, and a particular challenge was to identify a finite and manageable number of working groups where essentially all individuals within our broad neuroscience community could belong. We elected to establish 6 divisions within the IND which would be inclusive of members with common interests. Importantly, we wished to encourage further evolution of each of these groups, such that with additional resources and organization, the division may ultimately be elevated to 'Center' status.

- Disorders as a focus. While bench neuroscientists relish the idea of studying mechanisms of axoplasmic flow or neuronal phenotype maintenance for the sake of understanding the basic biology, the reality is that just

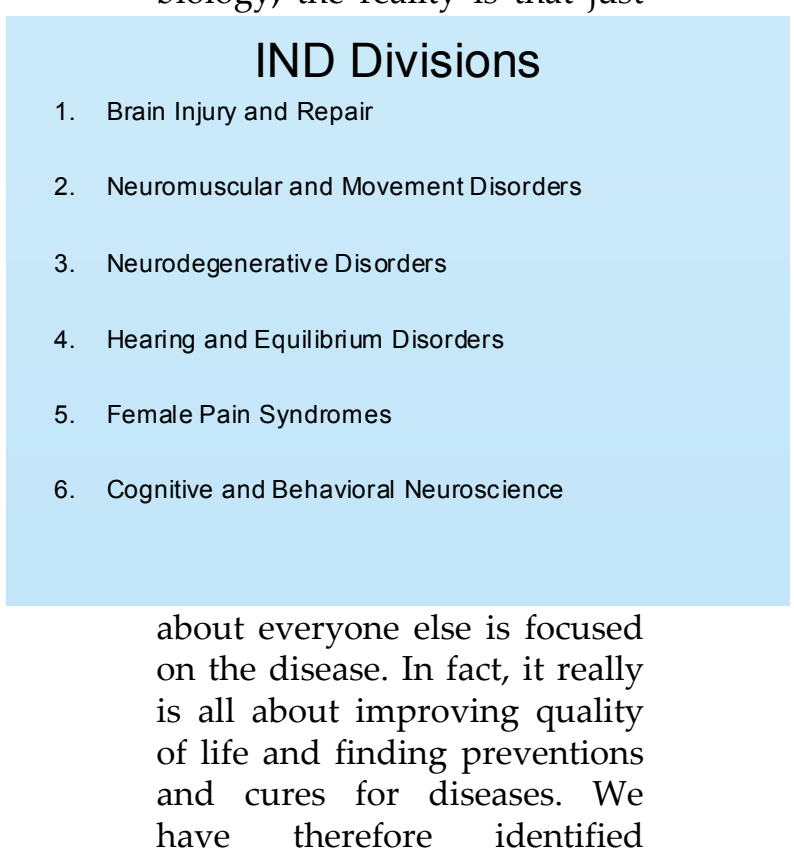

\section{Disease Focus Areas}

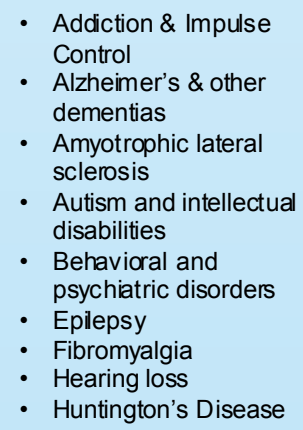

- Addiction \& Impulse Control

- Alzheimer's \& other dementias

- Amyotrophic lateral sclerosis

- Autism and intellectual disabilities

- Behavioral and psychiatric disorders

- Epilepsy

- Fibromyalgia

- Hearing loss

- Huntington's Disease

- Parkinson's Disease

- Pelvic pain

- Migraine \& TMJ

- Multiple Sclerosis

- Myasthenia Gravis

- Myopathies

- Spinal Cord Injury

- Stroke

- Tinnitus

- Traumatic Brain Injury

- Tremors

- Vertigo \& balance disorders
- Peripheral neuropathy specific diseases where we have sufficient expertise to justify the claim that a collaborative research team exists. Accordingly, our efforts are targeted toward some 22 specific disorders where such strengths exist.

With these defining principles in mind, the IND was organized and launched in March 2009, nearly a year after it was first conceived. Our primary 
objective was to advance neuroscience translational research programs regionally by coordinating and consolidating basic and clinical research. To do so, we identified 7 specific aims.

1. Provide administrative structure. Very little gets done without significant administrative input. By partnering with existing administrative units, it was possible for the IND to rapidly establish the administrative structure necessary to maintain communication among members and to coordinate events.

2. Promote interactions \& communication. As noted above, communication follows directly from having an administrative infrastructure in place. In addition, we've developed mechanisms that seek to promote communication among IND members (see discussion of Translational Discovery Forums below). Arguably, this may be the single most important element necessary for creating cohesion and collaboration.

3. Recruit researchers. The IND is playing a major role in the recruitment of neuroscience researchers. Perhaps the most significant advantage offered by the Institute offers is to bring together multiple partners working toward a common goal. In these days of limited resources, it is increasingly important that departments and centers who share similar needs work together to identify the mean necessary for successfully recruiting the right candidate. The IND has become an integral player in the neuroscience recruitment process, first by helping to identify the appropriate target recruitment area, and then by brokering arrangements whereby multiple departments and centers contribute resources toward the planned recruitment.

4. Promote core technologies. The IND can play a substantive role in promoting technologies within the neuroscience community in two important ways. First, its multiinstitutional composition puts it in an excellent position to promote and coordinate core technologies available throughout the Kansas City region. In addition, the IND can play a major role in identifying areas of technological deficiencies, and then mustering resources necessary to incorporate these technologies into existing or new cores.

5. Graduate programs enhancements. The IND, because of its organizational structure, serves as an adjunct to graduate education in the realms of both coursework and training programs. Our organizational structure into divisions is highly conducive to course development, and effectively provides a set of faculty who would be qualified to provide lectures in the related areas. Moreover, the IND with its extensive membership and access to core and individual laboratory technologies, is an attractive partner in formulating a predoctoral training program application and is likely to be viewed as a plus by a peer review committee. Because of its multi-institutional nature and communications web, neuroscientists throughout the region are made aware of new courses originating in 
conjunction with the IND and therefore are in a better position to inform their students of educational opportunities available to them, even if they are not currently attending the KUMC program.

6. Commercialization pipelines. The field of neuroscience is fertile ground for development of patents and commercial products. Through enhanced collaborations,

multidisciplinary interactions, and close ties with drug development programs and offices of intellectual property, the IND is well positioned to encourage commercialization of neuroscience-related drugs and devices.

7. Integrated space. Because the IND embraces members from 6 different institutions, the probability of ever consolidating all members in a single building is low. Nonetheless, having a single building dedicated to bringing together basic and clinical neuroscientists and that can be identified as the home of the IND would do well to advance the concept of the Institute.

Translational Discovery Forums: A vehicle for clinical-basic conversations

A significant challenge in promoting translational research programs is developing a means of communication among individuals with convergent interests. All too often, clinicians have limited exposure to basic scientists and do not attend common functions. While individuals may have similar interests, often times the clinical or basic researchers are unaware of ongoing related activities across the street. The objective in creating Translational Discovery Forums ( TDFs) was to provide a vehicle that would bring together established scientists and trainees, clinicians and basic researchers, to share interests and ideas in a setting that encourages interactions.

Our TDFs consist of interactions centered around a discipline or collaborative approach to a neurological disorder (a few of the topics to date include peripheral neuropathy, fibromyalgia, multiple sclerosis, Alzheimer's disease, and epilepsy). We typically hold these late Friday afternoon, which seems to be a time most compatible with the schedules of both clinicians and basic science faculty. The format is as follows:

- Clinical presentation. An overview of the disorder that forms the basis for the TDF is presented or, alternatively a case history or even patient presentations have formed the basis for this half-hour session. These are presented by a student, fellow or attending physician.

- Basic science journal presentation. This component integrates the established Neuroscience Journal Club. A student in the neurosciences selects a paper relevant to the disorder under discussion and presents this to the audience. The emphasis in this half-hour segment is to stress the relationship of the research to advancing our understanding of the disease.

- Collaborative research presentation. This forms the core of the TDF. Basic scientists and clinicians with common interests in a specific disorder present their research program relevant to the disease. They are strongly encouraged to describe ongoing collaborations and clinical-basic interactions or, if these are not in place, then the areas where 
collaborations could occur are described in this one-hour session. Bringing in members from different institutions is strongly encouraged.

These simple monthly forums have received a remarkable level of interest and turned out to be quite effective. Basic scientists frequently work on a disease process without fully understanding the clinical perspective, and therefore especially appreciate the introductory clinical overview. Similarly, clinicians often are not aware of how basic science can be brought to bear in investigating disease mechanisms or treatments, and are often surprised at how revealing some basic science publications may be to disease mechanisms. However, the greatest impact seems to be in the process of organizing the collaborative research presentation. Because these need to represent a coordinated effort, those presenting are encouraged to meet well before the scheduled TDF to discuss their presentations. In some cases where an existing collaboration is in place, there may be few surprises, and a history of how the collaboration evolved is instructive to those groups that are not as far along. However, in a number of cases these meetings have tended to be revelational, where obvious areas of collaboration emerge and light bulbs are turned on. Importantly, TDFs are open to anyone who wants to come, including patients with interest in the disorder. Accordingly, we encourage presenters to take a very basic approach and avoid the technical or jargon-laden tour de force approach, thus making these presentations more accessible to all in attendance.

\section{Partnerships with existing programs}

One area of collaborative evolution that has become increasingly important over the past decade or so is the extent to which independent programs must now partner with others. The pressures to partner were probably less evident in earlier times of more abundant resources, but it is now clear that an economy of scale can be beneficial. Typically, independent programs have common interests and needs, and there is little advantage in duplicating existing resources that may already have the capacity to serve additional purposes.

Given that the IND in many ways originated from within the Kansas IDDRC, it will not be surprising that these 2 entities are closely aligned and are partnering in areas of intersecting interest. However, the IND has also sought partnerships with other programs as well. These include:

- The Heartland Institute for Clinical and Translational Research. The HICTR serves as the primary regional instrument for advancing translational research in Kansas City, and serves as the organizing force behind an application for a Clinical and Translational Sciences Award. There are clearly a large number of intersecting objectives between the HICTR and IND, and the IND serves to organize neuroscience activities within the HICTR.

- The Kansas IDeA Network for Biomedical Research Excellence. This state-wide program funded by the NIH National Center for Research Resources is intended to promote educational and research programs, with emphasis on cell and developmental biology. There are 
several areas where their interests converge with those of the IND, particularly in regard to core services, and areas of common interest continue to evolve.

- Departments with strengths in neurosciences. Several departments have strong programs in the neurosciences and the IND is committed to working closely with these toward common goals. A particularly strong area of convergence pertains to recruitments. Because institutionally all faculty appointments are made through departments, the IND is highly dependent on close interactions in this regard. However, the IND has emerged as a particularly important player in recruitments by serving as a brokerage to bring together multiple players, as larger home for neuroscience recruits, and for providing assistance to clinical departments wishing to expand their research base.

- Center on Aging and the Hoglund Brain Imaging Center. The IND has much in common with other centers that support neuroscience clinical and basic activities. As with the departments, the IND has helped in recruitments and in organizing research programs and the Centers have provided access to programs and core technologies that have served to advance the regional neuroscience effort.

- The KU Endowment Association. Philanthropic support represents an increasingly important mechanism for promoting research programs. Private or foundation donations can support a number of important functions, including research starter funds for generating preliminary data prior to applying for NIH funding, named lectureships, scholarships and fellowships, endowed professorships, and institute or center directorships.

\section{Conclusions}

The IND, barely 6 months old at the time of this writing, remains a young and developing entity. Much remains to be done, and many challenges are yet to be overcome. However, the progress to date has been very encouraging. IND membership now exceeds 120 members, with membership in individual Divisions ranging from about 10 to 55 . About half of our members are clinicians or clinician-scientists. While the majority is located at KUMC, we have members from all major regional institutions. We have been successful in brokering one major recruitment and are exploring the possibility of a second. Our TDFs continue monthly, and are very highly attended. Clearly, the true metrics for success will come in the form of new collaborations leading to grants, papers, and other evidence of scientific advancement. Such outcomes take time, so we do not yet know the extent of the impact of the IND. Nonetheless, we have seen in several instances new collaborations arise, often unexpectedly and frequently across institutional boundaries. The IND therefore seems to be providing an effective vehicle for moving quickly into a new era of enhanced regional collaborations within the Kansas City area. 